\title{
Rucaparib Phosphate
}

National Cancer Institute

\section{Source}

National Cancer Institute. Rucaparib Phosphate. NCI Thesaurus. Code C78186.

The phosphate salt form of rucaparib, an orally bioavailable tricyclic indole and inhibitor of poly(ADP-ribose) polymerases (PARPs) 1 (PARP1), 2 (PARP2) and 3 (PARP3), with potential chemo/radiosensitizing and antineoplastic activities. Upon administration, rucaparib selectively binds to PARP1, 2 and 3 and inhibits PARP-mediated DNA repair. This enhances the accumulation of DNA strand breaks, promotes genomic instability and induces cell cycle arrest and apoptosis. This may enhance the cytotoxicity of DNAdamaging agents and reverse tumor cell resistance to chemotherapy and radiation therapy. PARPs are enzymes activated by single-strand DNA breaks that catalyze the post-translational ADP-ribosylation of nuclear proteins, which induces signaling and the recruitment of other proteins to repair damaged DNA. The PARP-mediated repair pathway plays a key role in DNA repair and is dysregulated in a variety of cancer cell types. 